\title{
Revisión de la Literatura sobre el Enfoque Estratégico de los Modelos de Negocios
}

\author{
Martha C. Ramírez ${ }^{(1)}{ }^{*}$, María del P. Rodríguez ${ }^{(2)}$ y José P. González ${ }^{(3)}$ \\ (1) Universidad Nacional de Colombia Sede Manizales, Campus La Nubia, Facultad de Ingeniería y Arquitectura, \\ Departamento de Ingeniería Industrial, Grupo de Investigación Ethos, Manizales-Colombia. \\ (e-mail: mcramiress@unal.edu.co) \\ (2) Universidad Nacional de Colombia Sede Manizales, Campus La Nubia, Facultad de Ingeniería y Arquitectura, \\ Departamento de Ingeniería Industrial, Grupo de Investigación Ethos, Manizales-Colombia \\ (e-mail: mdrodriguezco@unal.edu.co) \\ (3) Tecnológico Nacional de México en Celaya, Departamento de Ciencias Económico-Administrativas, Celaya, \\ Guanajuato, México. (e-mail: porfirio.gonzalez@itcelaya.edu.mx)
}

* Autor a quien debe estar dirigida la correspondencia

Recibido Mar. 8, 2019; Aceptado Abr. 25, 2019; Versión final May. 30, 2019, Publicado Dic. 2019

\section{Resumen}

Este artículo presenta un panorama de la literatura sobre enfoques estratégicos de los modelos de negocios. Su objetivo es proponer una clasificación para delimitar futuras líneas de trabajo que reconcilien las diferentes tendencias que presentan las investigaciones en este tema. La metodología empleada combina la búsqueda sistemática con la narrativa. La sistemática se realiza con la ayuda de la plataforma web Tree of Science, mientras que la narrativa se realiza a través de la consulta en las principales revistas que publican sobre el tema. Se identifican siete enfoques estratégicos: prescriptivo, cognitivo, contextual, del aprendizaje, cultural, sustentable e integrador. Se concluye que las perspectivas más consolidadas son la prescriptiva y del aprendizaje, seguidas de la contextual y sustentable. Las menos desarrolladas son la cognitiva, cultural e integradora. El enfoque sustentable requiere mayor investigación, ya que es reconocido como uno de los retos que enfrentan los negocios, perfilándose como una línea de investigación prometedora. estratégico

\section{Literature Review on the Strategic Approach of Business Models}

\begin{abstract}
This article presents an overview of the literature on strategic approaches of business models. Its objective is to propose a classification to delimit future lines of work that reconcile the different tendencies that the investigations in this area present. The methodology used combines the systematic search with the narrative. The web platform Tree of Science was used to carry out the systematic review, while the narrative search is done through consultation in the main journals that publish on the subject. Seven strategic approaches are identified: prescriptive, cognitive, contextual, learning, cultural, sustainable and integrative. It is concluded that the most consolidated perspectives are prescriptive and learning, followed by contextual and sustainable. The least developed approaches are the cognitive, cultural and integrative, emerging as promising lines of research. The sustainable approach requires additional research, since it is recognized as one of the challenges that businesses face, emerging as a promising line of research.
\end{abstract}

Keywords: strategy; business strategy; business model; Tree of Science; strategic approach 


\section{INTRODUCCIÓN}

Desde la década del 2000, la literatura sobre modelos de negocios (BM) se ha venido generando de manera progresiva con el impulso de revistas de corriente principal, tales como: Long Range Planning, Strategic Entrepreneurship Journal, Journal of Business Models y el Journal of Cleaner Production. Particularmente, se ha puesto de relieve que el enfoque estratégico es una de las disciplinas que han contribuido a su desarrollo (Maucuer y Renaud, 2019), y los asuntos estratégicos, son uno de los temas de mayor envergadura (Massa et al., 2017 y Zott et al., 2011). Desde esta disciplina, los BM son considerados un campo de investigación al lado de la estrategia, los cuales surgen como una nueva unidad de análisis que orienta acerca de la forma como se hacen los negocios frente a la incertidumbre, complejidad y dinamismo de los entornos. Se argumenta que estos agregan valor a la literatura sobre estrategia porque incluyen en la creación de valor, además de la oferta, a la demanda, desafiando los supuestos de las teorías tradicionales de la estrategia (Massa et al., 2017).

En virtud de lo expuesto, gran parte de las investigaciones sobre BM, desde la disciplina de la gestión estratégica, se han dedicado a estudiar la relación entre la estrategia y los BM, puesto que, su correspondencia conduce a una mayor competitividad, rendimiento, crecimiento y sostenibilidad de los negocios (Priem et al., 2018; Massa et al., 2017; Ladib y Lakhal, 2015; Zott et al., 2011). Empero, no se ha desarrollado una retórica clara que explique sobre los vínculos entre estos dos campos de investigación, dado que, se presentan diferentes posiciones. De un lado se especifica que los BM describen cómo interactúan los componentes de un negocio dentro de un sistema, pero no tienen en cuenta la competencia, el cual es un factor que afecta el rendimiento de los negocios; mientras que la estrategia, es formulada por las organizaciones para diferenciarse en términos de qué tipo de valor crear (Magretta, 2002). Bajo esta óptica se precisa que el BM es el marco para la ejecución de la estrategia (Cortimiglia et al., 2016; Galeano, 2014; Zott y Amit, 2010). Desde otro punto de vista se expone que los BM no necesariamente se derivan de una estrategia, aunque se reconoce que la relación entre estos dos elementos, es un factor condicionante para la competitividad de las organizaciones (Casadesus-Masanell y Ricart, 2010). Un planteamiento diferente, señala como una de las funciones del BM, la formulación de la estrategia competitiva (Chesbrough y Rosenbloom, 2002), y los asuntos estratégicos, como un componente importante del BM para la sostenibilidad de la ventaja competitiva (Morris et al., 2005).

En el trabajo de Stefanovic y Milosevic (2012), se argumenta que la estrategia y el BM son dos constructos inseparables y que la comprensión de sus diferencias y puntos de intersección, depende principalmente del punto de vista de la formación de la estrategia tomada por el autor. Así que en concordancia con el trabajo de Mintzberg y Waters (1985) comparten la visión de la estrategia como la fusión de intenciones gerenciales deliberadas, los esfuerzos posteriores de implementación y los desarrollos imprevistos emergentes y de aprendizaje. Desde esta perspectiva reactiva, la estrategia es un patrón dentro del cual un BM cambia. Pese a que se ha tratado de dar mayor claridad sobre la relación entre la estrategia y el BM, la extensa literatura desarrollada alrededor del tema presenta confusión sobre los vínculos que los unen (Wirts et al., 2016). De igual modo, aunque Maucuer y Renaud (2019) distinguen como subtemas, dentro de la disciplina de la gestión estratégica, a la internacionalización, los BM sociales, los procesos de creación de valor; señalan que estos asuntos no están bien fundamentados. Por tanto, hay una necesidad latente de desarrollar un trabajo que presente una visión integradora y, a la vez, permita contemplar la pluralidad de las perspectivas estratégicas que analizan los BM con el fin de brindar una mayor comprensión. Este vacío identificado, ha sido el fundamento para desarrollar la presente revisión literaria. El propósito de este artículo consiste en proponer una clasificación sobre los enfoques estratégicos que direccionan los BM, para lo cual, desde una posición integradora se realiza un estudio selectivo y crítico de la literatura que trata los temas de estrategia y BM simultáneamente, alrededor de patrones de comportamiento comunes y divergencias (Ramos et al., 2013; Velásquez, 2014).

Se decidió tomar como base el trabajo de Mintzberg et al. (1999) para la categorización, porque presenta una visión detallada sobre los diferentes trabajos que abordan el proceso de creación de la estrategia, los cuales agrupa en 10 escuelas (diseño, planificación, de posicionamiento, cognoscitiva, empresarial, aprendizaje, poder, cultural, ambiental, configuración) y aunque cada una se centra en aspectos diferentes, en su conjunto muestran un panorama más completo de la realidad. Los resultados de este estudio delimitan líneas de trabajo que se proponen como una base sólida sobre la cual otros autores pueden continuar construyendo nuevos conceptos teóricos; también, como directriz para investigaciones empíricas. Para cumplir con el objetivo propuesto, este artículo se estructura en tres secciones: la primera describe el método empleado para la revisión de la literatura, que combina la búsqueda narrativa con la sistemática, esta última se realiza a través de la plataforma web Tree of Science (Robledo et al., 2014). La segunda, presenta los resultados y discusión, dentro de los cuales se establecen como enfoques estratégicos que abordan los BM al prescriptivo, cognitivo, contextual, del aprendizaje, cultural, sustentable e integrador; además se expone un panorama sobre las 
corrientes de investigación de cada uno de los enfoques, en concordancia con las orientaciones teóricas que presentan los estudios, y sobre los posibles asuntos a tratar. Finalmente, se presentan las conclusiones.

\section{METODOLOGÍA}

Una revisión bibliográfica es un medio para identificar, organizar, evaluar e interpretar estudios relevantes para un área de interés o pregunta de investigación (Velásquez, 2014). Con el ánimo de dar respuesta al interrogante ¿Cuáles son las perspectivas estratégicas que se emplean para el estudio de los BM? se siguió la ruta metodológica ilustrada en la Figura 1 que se compone de tres etapas: búsqueda bibliográfica, análisis de contenido y clasificación, discusión y conclusiones.

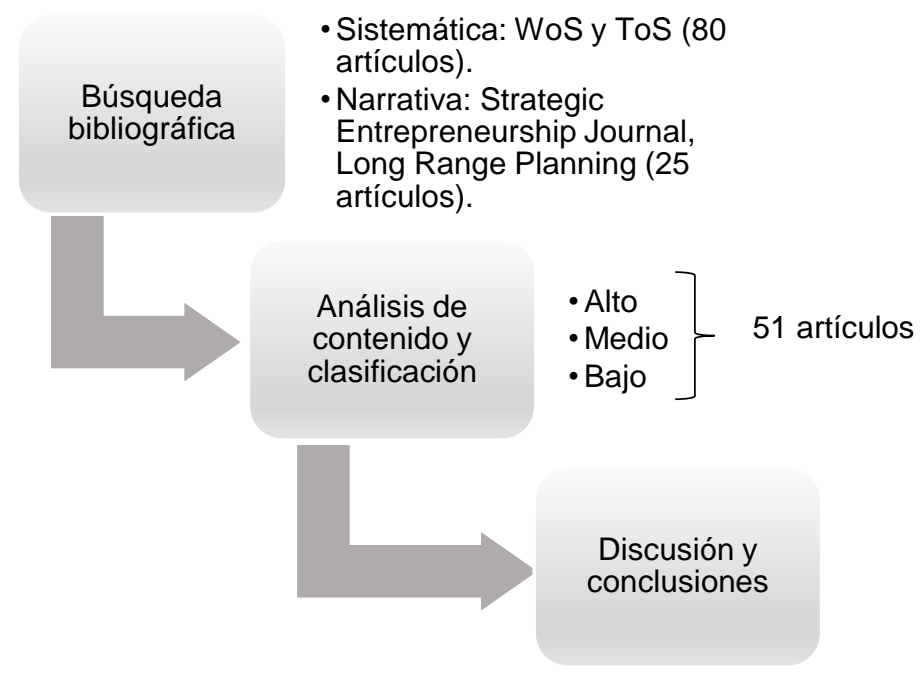

Fig.1: Ruta metodológica para responder a la pregunta central de la revisión bibliográfica

Para la búsqueda bibliográfica, se realizó una sinergia entre la búsqueda sistemática con la narrativa. La búsqueda sistemática utiliza una metodología definida para realizar la revisión de manera objetiva y hasta cierto punto replicable (kitchenham et al.,2004); en la búsqueda narrativa prima es el criterio subjetivo del revisor para acceder a los datos primarios y para integrar los resultados(Ferreras-Fernández, 2016). La articulación entre estas formas de revisión brinda un mayor panorama sobre el tema de interés y posibilita el análisis crítico de las investigaciones.

Como estrategia principal de búsqueda sistemática se utilizó la herramienta Tree of Science (ToS) que emplea la base de datos Web of Science (WoS), una de las colecciones de investigaciones científicas más ricas del mundo. El ToS fue desarrollado por Robledo et al. (2013), tiene sus bases en la teoría de grafos y permite, a través del análisis de redes de citaciones, identificar los artículos que tienen mayor relación con un área específica de conocimiento. Este análisis se realiza mediante la aplicación de tres criterios: 1) la cantidad de veces que se cita una referencia dentro de la red; 2) el número de veces que una referencia cita otras referencias y; 3) la probabilidad de que una referencia conecte otros dos elementos de la red seleccionados al azar. Las redes de citaciones se presentan en el ToS mediante la estructura de un árbol: raíces, tronco y hojas. Las raíces incluyen los estudios más citados, considerados como clásicos o pioneros. El tronco está formado por referencias que contribuyen a la consolidación de los enfoques derivados de los estudios clásicos. Las hojas pertenecen a los estudios más recientes, que van generando ramificaciones dentro de los diferentes enfoques (Diez et al., 2019; Toro y Rodríguez, 2017; Zuluaga et al., 2016 y Robledo et al., 2014).

La consulta en la WoS se hizo en abril de 2018, con la ecuación de búsqueda: Titulo: (strateg* AND business models) Timespan=All years. Databases=SCIEXPANDED, SSCI, A\&HCl. Para delimitar la ecuación se estipuló que los conceptos debían ser referenciados en el título de tal manera que solo se incluyeran artículos que tuviesen una relación directa entre estos. No se restringió el idioma y la fecha de publicación de las investigaciones para facilitar la identificación de los autores pioneros, (ver tabla 1). Esta búsqueda arrojó un total de 154 publicaciones, las cuales se exportaron a la plataforma ToS, que de manera automática y a través de la aplicación de su algoritmo de búsqueda y selección, generó un árbol con 80 artículos clasificados en 10 pioneros, 10 extensiones de los pioneros y 60 de nuevas vertientes de investigación. En la tabla 1 se presentan en orden cronológico los autores de los trabajos seleccionados en la búsqueda sistemática, agrupados de acuerdo con el ToS, en pioneros, extensiones de los pioneros y nuevas ramificaciones, y se marcan con * aquellos que se incluyen en la categorización de los enfoques estratégicos de los BM. 
Tabla 1: Artículos seleccionados en la búsqueda sistemática.

\begin{tabular}{|c|c|c|c|}
\hline \multicolumn{2}{|c|}{ Pioneros (10) } & \multicolumn{2}{|c|}{ Extensiones (10) } \\
\hline Amit y Zott $(2001)^{\star}$ & $\begin{array}{l}\text { Baden-Fuller y Morgan } \\
(2010)^{*}\end{array}$ & Zott C y Amit $(2008)^{*}$ & Bock et al. $(2012)^{\star}$ \\
\hline $\begin{array}{l}\text { Chesbrough y } \\
\text { Rosenbloom (2002)* }\end{array}$ & Chesbrough (2010) ${ }^{\star}$ & $\begin{array}{l}\text { Casadesus-Masanell y } \\
\text { Ricart }(2010)^{*}\end{array}$ & Hacklin y Wallnöfer (2012)* \\
\hline Magretta (2002)* & Demil y Lecocq (2010)* & Smith $(2010)^{*}$ & Achtenhagen et al. (2013)* \\
\hline Morris et al. (2005) ${ }^{\star}$ & Zott y Amit ( 2010)* & Teece $(2010)^{*}$ & Zott y Amit $(2013)^{*}$ \\
\hline $\begin{array}{l}\text { Osterwalder y Pigneur } \\
(2009)^{\star}\end{array}$ & Zott et al. (2011) & Aspara et al. (2011)* & Khanagha et al. (2014) \\
\hline \multicolumn{4}{|c|}{ Ramificaciones (60) } \\
\hline McAdam y Bailie (2002) & Liu y Wang (2010) & de Souza (2015) & Moutinho (2016) \\
\hline Bleistein et al. (2004) & Deodhar et al. (2012) & Gerpott (2015) & $\begin{array}{l}\text { Ocasio y Radoynovska } \\
(2016)^{\star}\end{array}$ \\
\hline $\begin{array}{l}\text { Freeman y Browne } \\
(2004)\end{array}$ & Karpen et al. (2012) & Gutierrez et al. (2015) & Spieth et al. (2016) \\
\hline Koo et al. (2004) & Leblebici. (2012) & Haubro et al. $(2015)^{\star}$ & Teymoori et al. (2016) ${ }^{\star}$ \\
\hline Lee et al. ( 2004) & Rajala et al. (2012) & $\begin{array}{l}\text { Ritala y Huizingh } \\
\text { (2015) }\end{array}$ & Vitasek (2016) \\
\hline $\begin{array}{l}\text { Toms y Filatotchev } \\
(2004)\end{array}$ & Sako (2012) & Saebi y Foss $(2015)^{\star}$ & $\begin{array}{l}\text { Al Humaidan y Sabatier } \\
(2017)^{\star}\end{array}$ \\
\hline Wang et al. (2004) & $\begin{array}{l}\text { Stefanovic y Milosevic } \\
(2012)^{*}\end{array}$ & Ahuja y Novelli (2016) & Arbussa et al. (2017) \\
\hline Burn y Ash (2005) & de Langen (2013) & Antoldi et al. (2016)* & Bonakdar et al. (2017) \\
\hline Tsai et al. (2005) & Huang y Chuang (2013) & $\begin{array}{l}\text { Bialek-Jaworska y } \\
\text { Gabryelczy (2016) }\end{array}$ & Franca et al. (2017)* \\
\hline $\begin{array}{l}\text { Pritchard y MacPherson } \\
\text { (2007) }\end{array}$ & $\begin{array}{l}\text { Osterwalder y pigneur } \\
\text { (2013) }\end{array}$ & Bini et al. (2016) & Kurucz et al. (2017) \\
\hline Seelos y Mair (2007) ${ }^{*}$ & Shavarini et al. (2013) & Bocken et al. (2016) ${ }^{*}$ & Manning et al. $(2017)^{\star}$ \\
\hline Hynes (2009) & Wei et al. (2013) & $\begin{array}{l}\text { Cortimiglia et al. } \\
(2016)^{\star}\end{array}$ & Bouwman et al. $(2018)^{\star}$ \\
\hline Murray (2009) & Yang y Su (2013) & Dellyana et al. (2016) & Kim y Chun (2018) \\
\hline Ricart (2009) & Cao (2014) & $\begin{array}{l}\text { Gäertner y Schön } \\
(2016)\end{array}$ & Priem et al. (2018) \\
\hline Kijl et al. (2010) & Horkoff et al. (2014) & Johnson y Foss (2016) & Visnjic et al. (2018) \\
\hline
\end{tabular}

A estos 80 artículos se sumaron otros 25 provenientes de una revisión narrativa que se realizó de forma manual en bases de datos, después de haber eliminado aquellos que también se encontraron en la revisión sistemática. Su objetivo era identificar trabajos sobre BM, en el campo de la gestión estratégica, en revistas de alto impacto, tales como: Long Range Planning (eg, Ammar y Chereau, 2018; Hacklin et al. 2018; Thompson y MacMillan, 2010; Yunus et al., 2010) y Strategic Entrepreneurship Journal (eg, Brea-Solis et al 2015; Kim y Min 2015; Martins et al., 2015 y Andries et al., 2013), también se encontraron estudios que, al ser muy citados dentro de las referencias de las publicaciones analizadas por ToS, son pertinentes para la revisión (eg, Badén-Fuller y Mangematin, 2013; Johnson et al., 2008; Chesbrough, 2006; Markides y Charitou, 2004; Prahalad y Hart, 2002). En la tabla 2 se presentan en orden cronológico los autores de los trabajos seleccionados en la búsqueda narrativa y se resaltan con * aquellos que se incluyen en la categorización de los enfoques estratégicos de los BM.

Después de una revisión preliminar del total de artículos, se identificaron 4 revisiones literarias (Maucuer y Renaud, 2019; Massa et al., 2017; Wirts et al., 2016; Zott et al., 2011) que no se incluyen dentro de la clasificación de los enfoques estratégicos de los BM, aunque contribuyeron a orientar la presente revisión. Seguidamente, se realiza un estudio selectivo y crítico según el nivel de relación que tienen con la pregunta guía de la revisión bibliográfica ¿Cuáles son las perspectivas estratégicas que se emplean para el estudio de 
los BM? lo cual permitió clasificar los estudios, por orden de relevancia en alto, medio y bajo, Al final se llegó a un total de 51 artículos, de los cuales $31(61 \%)$ provienen de la revisión sistemática y $20(39 \%)$ de la narrativa. La información obtenida de los artículos se estandarizó con base en los siguientes criterios: 1) objetivo de la investigación; 2) relaciones de la estrategia de negocios con el BM; 3) enfoques estratégicos implícitos o explícitos de los estudios, tomando como referencia el trabajo de Mintzberg (1999) y, 3) resultados y futuras líneas de investigación.

Tabla 2: Artículos seleccionados en la búsqueda narrativa.

\begin{tabular}{|c|c|c|c|}
\hline Prahalad y Hart (2002) & Yunus et al. (2010)* & Brea-Solis et al. (2015) & Massa et al. (2017) \\
\hline $\begin{array}{l}\text { Markides y Charitou } \\
(2004)^{\star}\end{array}$ & $\begin{array}{l}\text { Cavalcante et al. ( } \\
2011)^{*}\end{array}$ & Demil et al. $(2015)^{*}$ & Hacklin et al. (2018)* \\
\hline Chesbrough (2006) & Leblebici H (2012) & Goyal et al. ( 2015)* & Tallman et al. (2018)* \\
\hline Johnson et al. (2008)* & Andries et al. (2013)* & Kim y Min (2015)* & $\begin{array}{l}\text { Maucuer y Renaud } \\
\text { (2019) }\end{array}$ \\
\hline Mason y Leek (2008)* & $\begin{array}{l}\text { Badén-Fuller y } \\
\text { Mangematin }(2013)^{*}\end{array}$ & Martins et al. ( 2015) & \\
\hline O'reilly $(2009)^{*}$ & Markides $(2013)^{*}$ & $\begin{array}{l}\text { Christensen et al. } \\
(2016)^{\star}\end{array}$ & \\
\hline $\begin{array}{l}\text { Thompson y MacMillan } \\
(2010)^{*}\end{array}$ & Barki et al. (2015)* & Wirts et al. (2016) & \\
\hline
\end{tabular}

\section{RESULTADOS Y DISCUSIÓN}

Los estudios que abordan la relación entre estrategia y BM muestran que las perspectivas estratégicas de la posición competitiva (Porter, 1985), la visión basada en los recursos de la firma - VBR - (Barney, 1991) y el enfoque relacional (Dier y Sing, 1998), se han venido integrando para explicar el funcionamiento de los BM. Desde esta óptica, el análisis de los BM va más allá de la configuración de la cadena de valor, la explotación de competencias centrales específicas de la empresa y el enfoque de redes estratégicas entre empresas (Amit y Zott, 2001). Así, el BM es una descripción de como una organización funciona para lograr sus objetivos de crecimiento, rentabilidad e impacto social (Massa et al., 2017).

Si bien la lógica de los negocios que plantea Porter (1985) se fundamenta en la concurrencia de múltiples competidores que optan por diferentes posiciones estratégicas (liderazgo en costos o diferenciación), en procura de una ventaja superior frente a sus rivales, también es cierto que las fuerzas básicas de la industria impulsan las relaciones de la empresa con otros agentes que intervienen en el negocio para este mismo fin. Dentro de este marco de ideas, el BM es definido como la construcción de un sistema de actividades que va más allá de los límites de la organización, dando como resultado negocios más competitivos (Zott y Amit, 2010). De otro lado, la teoría de la VBR señala que la combinación de recursos y capacidades de una organización son fuente de creación de valor y de ventaja competitiva, pues la interacción con otros agentes del mercado puede producir una oferta de valor única para satisfacer las necesidades del cliente y constituirse en una de las barreras contra la imitación del BM, debido a la complejidad de las relaciones (Teece, 2010). Ahora bien, desde la perspectiva relacional de la estrategia, la creación de valor en las redes se refiere al ajuste de las capacidades distintivas que van más allá de los recursos y las capacidades que se encuentran entre los límites de la organización (Dyer y Singh, 1998).

Lo anterior ilustra cómo diferentes frentes de la estrategia están convergiendo para explicar la complejidad de los BM. Asimismo, cómo la comprensión de las diferencias y puntos de intersección entre la estrategia y el modelo de negocio, depende del punto de vista de la formación de la estrategia tomada por el autor (Stefanovic y Milosevic, 2012). Bajo este horizonte y tomando como base el trabajo de Mintzberg et al. (1999), el proceso de evaluación de contenidos de cada estudio permitió clasificar los enfoques estratégicos de los BM, en siete categorías: prescriptivo, cognitivo, contextual, del aprendizaje, cultural, sustentable e integrador. Cabe aclarar al respecto, que en este trabajo al enfoque ambiental se le denomina contextual para evitar confusiones con la orientación sustentable de los negocios, el cual se incluye como una nueva perspectiva estratégica, por la necesidad latente que tienen las organizaciones de diseñar BM que contribuyan a resolver problemas de índole económico, social y ambiental. Además, hay que precisar que, aunque los trabajos analizados tienen un enfoque particular, la mayoría de ellos tratan de manera tangencial otras perspectivas, lo cual deja notar su complementariedad. En la Tabla 1 se presenta los 51 artículos objeto de estudio, agrupados de acuerdo con el ToS, en pioneros (14), extensiones de los pioneros (16) y nuevas ramificaciones (21). Además, se clasifican según los enfoques referidos tanto como corriente central (identificados con $\mathbf{x}^{*}$ ) como complementarios (señalados con $\mathrm{x}$ ). 
Tabla 3: Lista de artículos clasificados según los siete enfoques estratégicos de los BM.

\begin{tabular}{|c|c|c|c|c|c|c|c|c|}
\hline Autores & Año & Prescriptivo & Cognitivo & Contextual & Cultural & Aprendizaje & Sustentable & Integrador \\
\hline \multicolumn{9}{|c|}{ 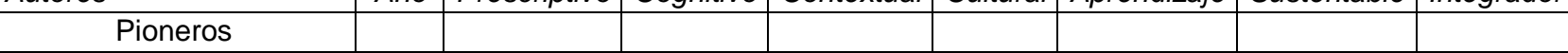 } \\
\hline Amit y Zott. & 2001 & $\mathrm{X}^{\star}$ & & & & & & \\
\hline $\begin{array}{l}\text { Chesbrough y } \\
\text { Rosenbloom. }\end{array}$ & 2002 & & $\mathrm{X}$ & $\mathrm{X}^{*}$ & & $\mathrm{X}$ & & \\
\hline Magretta. & 2002 & $\mathrm{X}^{\star}$ & $\mathrm{X}$ & $\mathrm{X}$ & & & & \\
\hline Markides y Charitou. & 2004 & & & & & $\mathrm{X}^{*}$ & & \\
\hline Morris et al. & 2005 & $\mathrm{X}^{\star}$ & $\mathrm{X}$ & $\mathrm{X}$ & & & & \\
\hline Chesbrough. & 2006 & $\mathrm{X}$ & & & & $\mathrm{X}^{*}$ & & \\
\hline Johnson et al. & 2008 & $\mathrm{X}^{\star}$ & & $\mathrm{X}$ & & & & \\
\hline Mason y Leek. & 2008 & & & & & $\mathrm{X}^{*}$ & & \\
\hline Osterwalder y Pigneur. & 2009 & $\mathrm{X}^{*}$ & & & $\mathrm{X}$ & & $\mathrm{X}$ & \\
\hline Badén-Fuller y Morgan. & 2010 & $\mathrm{X}^{\star}$ & $\mathrm{X}$ & & & & & \\
\hline Chesbrough. & 2010 & & & $\mathrm{X}$ & $\bar{X}$ & $X^{*}$ & & \\
\hline Demil y Lecocq. & 2010 & $\mathrm{X}^{\star}$ & $\mathrm{X}$ & & & & & \\
\hline Thompson y MacMillan. & 2010 & & & & & & $\mathrm{X}^{*}$ & \\
\hline Zott y Amit. & 2010 & $\mathrm{X}^{*}$ & & & & & & \\
\hline \multicolumn{9}{|l|}{ Extensiones } \\
\hline Zott y Amit. & 2008 & & & $\mathrm{X}^{*}$ & & & & \\
\hline O'reilly. & 2009 & & & & & $\mathrm{X}^{*}$ & & \\
\hline $\begin{array}{l}\text { Casadesus-Masanell y } \\
\text { Ricart. }\end{array}$ & 2010 & $X^{*}$ & $\mathrm{x}$ & $\mathrm{x}$ & & $\mathrm{X}$ & & \\
\hline Smith et al. & 2010 & & $\mathrm{X}$ & $\mathrm{X}^{*}$ & & $\mathrm{X}$ & & \\
\hline Teece. & 2010 & $X^{\star}$ & $\mathrm{X}$ & $\mathrm{X}$ & & $\mathrm{X}$ & & \\
\hline Yunus et al. & 2010 & $\mathrm{X}$ & & & & & $\mathrm{X}^{\star}$ & \\
\hline Cavalcante et al. & 2011 & & $\mathrm{X}$ & & & & & $\mathrm{X}^{*}$ \\
\hline Achtenhagen et al. & 2013 & & & & & $\mathrm{X}^{*}$ & & \\
\hline Andries et al. & 2013 & & & & & $\mathrm{X}^{*}$ & & \\
\hline $\begin{array}{l}\text { Baden-Fuller y } \\
\text { Mangematin. }\end{array}$ & 2013 & & $\mathrm{X}^{*}$ & & & & & \\
\hline Markides. & 2013 & & & & & $\mathrm{X}^{*}$ & & \\
\hline Khanagha et al. & 2014 & & & $\mathrm{X}^{*}$ & & & & \\
\hline Demil et al. & 2015 & & & $\mathrm{X}^{*}$ & & & & \\
\hline Martins et al. & 2015 & & $\mathrm{X}^{*}$ & & & & & \\
\hline Christensen. & 2016 & & & & & $\mathrm{X}^{*}$ & & \\
\hline Cortimiglia et al. & 2016 & & & $\mathrm{X}$ & & $\mathrm{X}^{*}$ & & $\mathrm{X}$ \\
\hline \multicolumn{9}{|l|}{ Ramificaciones } \\
\hline Seelos y Mair. & 2007 & & & & & & $\mathrm{X}^{*}$ & \\
\hline Aspara et al. & 2011 & & $\mathrm{X}^{*}$ & & & $\mathrm{X}$ & & \\
\hline Bock et al & 2012 & & & & $\mathrm{X}^{*}$ & $X$ & & \\
\hline Hacklin y Wallnöfer. & 2012 & & & & & $\mathrm{X}^{*}$ & & \\
\hline Stefanovic y Milosevic. & 2012 & & & & & $X^{*}$ & & \\
\hline Barki et al. & 2015 & & & & & & $\mathrm{X}^{*}$ & \\
\hline Brea-Solis et al. & 2015 & & & & & & & $\mathrm{X}^{*}$ \\
\hline Goyal et al. & 2015 & & & & & & $\mathrm{X}^{*}$ & \\
\hline Haubro et al. & 2015 & $\mathrm{X}^{*}$ & & & & & & \\
\hline Kim y Min. & 2015 & & & $\mathrm{X}^{*}$ & & & & \\
\hline Saebi y Foss. & 2015 & & $\mathrm{X}^{*}$ & & & $\mathrm{X}$ & & $\mathrm{X}$ \\
\hline Antoldi et al. & 2016 & $\mathrm{X}$ & $X^{*}$ & & & & & \\
\hline Bocken et al. & 2016 & & & & & & $\mathrm{X}^{*}$ & \\
\hline Ocasio y Radoynovska. & 2016 & & $\mathrm{X}^{*}$ & & & & & \\
\hline Teymoori et al. & 2016 & & & $\mathrm{X}^{*}$ & & & & \\
\hline Al Humaidan y Sabatier. & 2017 & & $\mathrm{X}$ & $\mathrm{X}^{*}$ & & & & \\
\hline Franca et al. & 2017 & & & & & & $\mathrm{X}^{*}$ & \\
\hline Manning et al. & 2017 & & & & & & $\mathrm{X}^{*}$ & \\
\hline Bouwman et al. & 2018 & $\mathrm{X}^{\star}$ & & & & & & \\
\hline Hacklin et al. & 2018 & & & & & $\mathrm{X}^{*}$ & & \\
\hline Tallman et al. & 2018 & & & & & & & $\mathrm{X}^{*}$ \\
\hline
\end{tabular}




\section{Enfoque prescriptivo de los BM}

Los estudios que se abordan desde este enfoque (ver Tabla 1) coinciden en sostener que: 1) la articulación de la estrategia con el BM es un factor fundamental para la creación de la ventaja competitiva y la sostenibilidad de los negocios, no obstante, los BM están sujetos a pruebas del mercado que los obliga a realizar ajustes en sus propuestas de valor y a una constante innovación; 2) la eficiencia de los costos de transacción, la creación de lazos duraderos con los clientes y las complementariedades entre los socios son las principales fuentes de creación de valor; 3 ) los BM son, por sí mismos, fuente de innovación y de ventaja competitiva y; 4) los directivos son protagonistas en el diseño de los BM. Estas aserciones han generado el surgimiento de otros enfoques, como el cognitivo, el contextual y el del aprendizaje.

El funcionamiento de los BM se aborda desde dos corrientes: los componentes de los BM como modelos a seguir y las dimensiones particulares de los BM. Para su explicación se han incorporado las teorías de la estrategia, los sistemas, la contingencia, las capacidades dinámicas y la ecología de las organizaciones. En La primera corriente, desde la orientación competitiva, se resalta la importancia de la participación de socios estratégicos en el negocio, con el fin de estructurar un sistema de actividades para crear valor (Zott y Amit, 2010). La orientación de la VBR, argumenta que el BM debe ser examinado a la luz de los filtros que debe superar una estrategia catalogada como exitosa para asegurar una ventaja competitiva: 1) segmentación del mercado; 2) creación de una proposición de valor para cada segmento; 3) diseño e implementación de mecanismos para capturar valor de cada segmento y; 4) implementación de mecanismos de aislamiento para obstaculizar la imitación por parte de los competidores y la desintermediación por parte de los clientes (Teece, 2010).

Estas concepciones han recibido grandes críticas por la orientación estática que ofrecen de los BM; lo cual, estimula la orientación dinámica de los BM que se produce por los cambios del entorno, así que, desde la teoría del ajuste estratégico, se propone armonizar la relación entre las dos orientaciones. Dentro de este marco de ideas, el concepto estático de los BM puede ayudar a identificar sus componentes centrales, pero las transformaciones del ambiente y la importancia de mantener la competitividad, alientan a las empresas a asumir nuevos BM o reconfigurar su sistema de actividades, provocando la evolución de los negocios (Antoldi et al., 2016; Demil y Lecoq, 2010). La teoría de sistemas también contribuye a explicar el dinamismo de los BM y su proceso de innovación. De acuerdo con este planteamiento, el BM es una representación concisa de un conjunto interrelacionado de variables de decisión a nivel económico (la lógica de la generación de ganancias), operativo (los procesos internos y el diseño de la infraestructura que permite a la empresa crear valor) y estratégico (la ventaja competitiva y la sostenibilidad). Así que un cambio que se produzca en uno de los componentes de los BM, puede alterar el funcionamiento de los demás elementos (Morris et al., 2005).

Es importante resaltar que el modelo Canvas, propuesto por Osterwalder y Pigneur (2009), es el más referido de los estudios prescriptivos. Éste, además de contener las corrientes teóricas anteriores, involucra herramientas de análisis estratégico para la evaluación de los BM. Bajo el Canvas, el BM se describe como el anteproyecto de la estrategia, proponiendo un esquema que divide el BM en nueve módulos interrelacionados, los cuales cubren las cuatro áreas principales de un negocio: clientes (segmento del mercado, canal, relaciones con el cliente), oferta (propuesta de valor), infraestructura (recursos clave, actividades clave, asociaciones clave) y viabilidad económica (estructura de costos y fuentes de ingresos). Adicionalmente, con el ánimo de sustituir aquellos BM que no crean valor para la empresa, el cliente y la sociedad, se sugiere el esquema de las cuatro acciones propuesto por Chan kim y Mourbogne (2005) y la matriz de análisis interna y externa (FODA) como herramientas de análisis. La primera herramienta, favorece tanto la generación de valor, como la disminución de costos; la segunda, contribuye a la comprensión de los cambios del entorno. Desde esta orientación, los cambios que se introducen en la propuesta de valor, tienen implicaciones directas en los demás módulos que integran el BM.

En la segunda corriente, desde el enfoque biológico, se propone un punto intermedio entre las teorías del Taylorismo, el Institucionalismo y la Teoría de Juegos. Las dos primeras, han tratado de interpretar la conducta de las organizaciones mediante roles ideales o modelos a escala. La última, explica su comportamiento de acuerdo con las posibilidades estratégicas y sus opciones. Según esta corriente, las organizaciones se comportan como organismos vivientes que experimentan cambios, los cuales las lleva a reafirmar o reinventar sus BM (Badén-Fuller y Morgan, 2010). Una propuesta similar se presenta a partir de la teoría de la estrategia, para analizar la singularidad de los BM, la cual también ha tenido una gran aceptación en el campo de los estudios empíricos, porque facilita la interpretación de BM complejos. En este planteamiento se hace énfasis en la importancia de diferenciar los conceptos de estrategia, BM y táctica para explicar el dinamismo de los negocios. La estrategia, se define como un plan de acción contingente que precisa el modelo de negocio a emplear con el fin de crear valor. Mientras que el BM está condicionado por la estrategia e incorpora el conjunto de elecciones (políticas, activos y estructuras de gobierno) y sus consecuencias derivadas, con el fin de cumplir con los objetivos propuestos. Por su parte, las tácticas son elecciones que se toman en relación 
con la estrategia y los límites establecidos por el BM. Estas últimas, se ocupan de determinar la forma con la que se compite en el mercado y por tanto, desempeñan un papel central en la creación de valor (CasadesusMasanell y Ricart, 2010).

\section{Enfoque cognitivo de los BM}

Los estudios clasificados bajo este enfoque (Tabla 1), destacan el papel protagónico de los directivos en el diseño y evolución de los BM, desde la perspectiva de pensamiento y acción. Bajo esta corriente, los directivos interpretan los cambios del entorno y van creando estructuras y procesos mediante los que se compite. En consecuencia, uno de los componentes básicos del BM son los aspectos cognitivos, donde las experiencias, capacidades y sistema de valores de los gerentes representan un papel protagónico en la creación y evolución de los BM (Saebi y Foss, 2015; Aspara et al., 2011). Así, el directivo es quien se ocupa de combinar de forma particular los componentes estratégicos básicos de los BM o de incorporar nuevos elementos que repercuten en innovaciones de los BM (Baden-Fuller y Mangematin, 2013). Por ser la mente de los estrategas el principal arquitecto de los BM, éstos pueden presentar limitaciones en las manifestaciones cognitivas de la evolución de los negocios y correr el riesgo de ser manipulados. Por ello, en este enfoque, se introduce el estudio de la dimensión psicológica del individuo.

Uno de los principales desafíos que enfrenta este enfoque radica en la dificultad para cambiar los esquemas cognitivos, debido a la resistencia al cambio que experimentan los directivos. Para responder a él, Martins et al. (2015) y Cavalcante et al. (2011), desde los planteamientos teóricos del razonamiento analógico y la combinación conceptual, argumentan que puede provocarse un giro en el que se rompe con las rutinas existentes y se evoluciona hacia una búsqueda activa de nuevas alternativas, aún en ausencia de cambios exógenos. A este respecto, Ocasio y Radoynovska (2016) advierten que cuando se trata de articular lógicas expuestas como incompatibles o paradójicas, para hacer frente a los cambios del mercado, se generan resultados diferenciados en relación con las transformaciones en los BM. En síntesis, un papel clave de los gerentes, consiste en modificar cuidadosamente el BM en el tiempo, con el fin no solo de asegurar un desempeño económico adecuado, sino también de lograr el objetivo de crear valor para el cliente (Antoldi et al., 2016).

\section{El enfoque contextual de los BM}

En los trabajos que abordan este enfoque (Tabla 1), se diferencian dos orientaciones de los BM según la posición que se tome en relación con el ambiente: determinista y voluntarista (Astley y Van de Ven,1983). Estas orientaciones han generado un cambio paradigmático de la perspectiva estratégica que conlleva al ajuste estratégico de los BM, en correspondencia con las dos posiciones (Demil y lecoq, 2010). Desde la orientación determinista, existen factores contingenciales (aspectos del entorno, estructura organizacional y tecnología) que pueden alterar el desempeño de la organización. En virtud de ello, se visualiza la estrategia como un proceso reactivo, lo cual indica que debe ser reformulada con la intención de contrarrestar las contingencias del entorno. Lo mismo ocurre con el BM, el cual se debe ajustar de acuerdo a los cambios del contexto, con el fin de alcanzar un mayor rendimiento. En tal sentido, la diferencia entre el éxito y el fracaso de las actividades de transformación se reduce a la capacidad para cambiar el BM de manera efectiva y en consonancia con la dinámica del ambiente (Cortimiglia et al., 2016; Aspara et al., 2011). La orientación voluntarista, se diferencia de la determinista en que la estrategia se trata como un proceso evolutivo que depende de las percepciones de los gerentes sobre lo que debería ser y alcanzar la organización (Wittington, 1988). En las dos orientaciones se afirma que aunque la estrategia y los BM, por separado, tienen la facultad para generar mayor competitividad, la interacción entre ambos genera un impacto mayor sobre los resultados empresariales (Zott y Amit, 2008; Casadesus-Masanell y Ricart, 2010).

Uno de los aspectos tratados en los estudios con enfoque contextual, consiste en analizar cómo las organizaciones afrontan situaciones de disolución ambiental, entendida esta como la segregación gradual de un nicho ecológico de negocios (Cameron y Zamuto, 1983). Conforme con esta presunción, las organizaciones incursionan en procesos de renovación estratégica, que implican la modificación o reemplazo de los BM cuando se visualizan oportunidades ambientales emergentes o se enfrentan con amenazas de supervivencia (Smith et al., 2010). Aquí, la renovación estratégica de los BM se entiende como un proceso dinámico que está sujeto tanto a la acción gerencial como a las fuerzas ambientales, a lo que se denomina "coevolución" (Flier et al., 2003).

Como segunda corriente de investigación se identifica la innovación en los BM como una de las líneas de investigación activas, al lado de producto, proceso y mercadeo (Chesbrough, 2006). De acuerdo con este planteamiento, las posibilidades de innovación en los BM dependen de la visión del entorno que tenga la administración. Así que los directivos con una orientación hacia la organización, probablemente, percibirán la disolución del medio ambiente como una amenaza y participarán en actividades de renovación incremental. 
En tanto, aquellos con una orientación hacia el mercado, la verán como una oportunidad y se involucrarán en actividades de renovación discontinua. Por su parte, los directivos con una orientación tecnológica, se inclinarán por generar transformaciones disruptivas (Al Humaidan y Sabatier., 2017). En general, la puesta en práctica de alternativas estratégicas introduce transformaciones en los BM que, en comparación con los procesos de innovación, comprenden un rango de posibilidades tales como: la evolución incremental, la transformación dirigida y la sustitución radical. Por consiguiente, a razón de una posible disolución del BM, las organizaciones apuntan al cumplimiento de tres objetivos estratégicos: 1) trabajar con el BM actual, al tiempo que experimenta con uno nuevo; 2) preparar la organización para una transición rápida y oportuna y; 3) combatir con los costos del cambio (Khanagha et al., 2014).

\section{El enfoque cultural de los BM}

Este enfoque es abordado por un solo artículo como corriente central y por otros dos que lo visualizan como complementario (ver Tabla 1). Aquí se hace énfasis en la creación de la estrategia como un proceso de interacción social basado en las convicciones e interpretaciones compartidas de manera tacita por los miembros de una organización. La cultura creativa reduce la resistencia al cambio y tiene un efecto positivo sobre la flexibilidad estratégica que consiste en la capacidad de identificar oportunidades de innovación, comprometer o reorientar recursos alrededor de los nuevos cursos de acción, con el fin de adaptarse rápidamente a los cambios del entorno que pueden afectar la posición competitiva de las organizaciones (Bock et al., 2012). De igual modo, se afirma que la cultura es uno de los principales factores que puede restringir el florecimiento de negocios emergentes, pues en ocasiones las costumbres y las normas establecidas limitan la posibilidad de aprovechar nuevas oportunidades de negocios; asimismo, puede interponer barreras a la innovación de los BM (Chesbrough, 2010; Osterwalder y Pigneur, 2009).

\section{El enfoque del aprendizaje de los BM}

Bajo este enfoque, la creación de la estrategia es vista como un proceso de aprendizaje social (Mintzberg et al., 1999) en el que los directivos de nivel estratégico centran la atención en el rol que desempeñan los administradores de nivel táctico y operativo. A nivel táctico, aquellos planteamientos estratégicos que realizan algunas unidades de negocios con perspectivas de mayor credibilidad, crecimiento y rentabilidad influencian la orientación competitiva de la organización y, por consiguiente, direccionan los cambios en el BM (Aspara et al., 2011). A nivel operativo, los directivos de base son la fuerza impulsora en la percepción y comprensión de oportunidades, apoyados en nuevas capacidades que aún no son reconocidas como distintivas. En tal sentido, el BM es una herramienta que puede ser utilizada para plantear estrategias de abajo hacia arriba porque representan un rol simbólico que facilita la comunicación, comprensión y ayuda a compartir el discurso estratégico (Hacklin y Wallnöfer, 2012). Dentro de este planteamiento (Tabla 1), se destacan cuatro orientaciones: 1) la estrategia como práctica; 2) las capacidades dinámicas; 3) el riesgo derivado y; 4) la visión ecológica.

En la primera orientación, la estrategia pasa de ser vista como un proceso de pensamiento y acción, a ser entendida como un proceso continuo y racional que se compone de planes deliberados, patrones de conducta y estrategias emergentes, lo cual hace que las organizaciones avancen mientras aprenden. Desde esta perspectiva, el BM es definido como una bisección de la estrategia, lo cual significa que la estrategia se presenta como un patrón de comportamiento dentro del cual el BM cambia. Por tanto, no se puede explicar como algo estático en el tiempo (Stefanovic y Milosevic, 2012). Esta orientación facilita la configuración, adaptación y renovación de los BM según las oportunidades del entorno (Achtenhagen et al., 2013).

En la segunda orientación, la habilidad de las organizaciones para cambiar su BM se ve influenciada por innovaciones que son el resultado de la renovación continua de las capacidades internas de la organización (Saebi y Foss, 2015). Por tanto, la generación de dinámicas positivas en los negocios (círculos virtuosos) motiva el fortalecimiento de las capacidades que son críticas en la creación de valor, lo cual provoca la evolución de los BM (Baden-Fuller y Mangematin, 2013; Casadesus-Masanell y Ricart, 2010). En ambientes inciertos y complejos es conveniente reconfigurar y enriquecer constantemente las capacidades ante la urgencia de responder a las contingencias. Así que los cambios que se realizan al BM existente pueden generar un mayor rendimiento que tratar con negocios paralelos porque posibilita la concentración de recursos críticos y facilitan la identificación, transformación y desarrollo de capacidades necesarias para hacer frente a las nuevas condiciones del entorno (Hacklin et al., 2018; Achtenhagen et al., 2013). Sin embargo, la evolución de las capacidades impulsada por los recursos que son estratégicos limitan las posibilidades de encontrar o adaptarse a nuevas oportunidades y desviar la atención gerencial en actividades que no son esenciales (Bock et al., 2012). En consecuencia, la experiencia y el conocimiento adquirido se convierten en un obstáculo para capturar el valor de las nuevas tecnologías, por lo cual se requiere diseñar nuevos BM que permitan gestionar estas innovaciones de manera acertada. Desde este ángulo, la innovación del modelo de negocio se convierte en una capacidad dinámica en lugar de ser el resultado de un proceso evolutivo de las capacidades, que 
puede llegar a cambiar la forma de competir de una industria (Christensen, 2016; Chesbrough, 2010; Chesbrough y Rosenbloom, 2002).

En la tercera orientación, se tiene en cuenta el riesgo derivado de la gestión simultánea de negocios duales, puesto que puede desatar la competencia por recursos internos, impedir el surgimiento de nuevos negocios con altas probabilidades de crecimiento o provocar el desplome anticipado de los existentes (Markides y Charitou, 2004). En este sentido, la incorporación de un nuevo negocio es conveniente para la mejora del desempeño organizacional solo en aquellos casos donde los activos de mayor relevancia tienen una función complementaria con el BM actual. Por el contrario, si dichos activos entran en conflicto resulta más conveniente crear un negocio autónomo para el reciente BM (Kim y Min, 2015). Otra situación que se aborda desde la teoría de riesgo consiste en el direccionamiento de estrategias paradójicas (refiriéndose a la capacidad de adoptar y gestionar estrategias que han sido consideradas como incompatibles), las cuales generan modelos de negocio complejos que resultan de las tensiones de su gestión (Smith et al., 2010). Esta situación hace que las organizaciones sean más resistentes en ambientes turbulentos al tiempo que desarrollan capacidades para responder creativamente a los cambios que se presentan.

En la cuarta orientación, se argumenta que solo sobreviven aquellos negocios que logren una mejor adaptación a los cambios tecnológicos y del mercado. Por consiguiente, la ambidiestralidad (capacidad que tienen las organizaciones de explotar sus negocios existentes y explorar nuevas oportunidades de negocios simultáneamente) promueve el aprendizaje continuo y, a su vez, motiva la dinamización de las capacidades que contribuyen a la evolución de los BM. Así que solo las compañías que tienen la posibilidad de modificar, crear y eliminar negocios sin poner en peligro su existencia tienen mayor oportunidad de sostenerse en el tiempo (O'Reilly III et al., 2009; Johnson et al., 2008). Desde esta óptica, los negocios de explotación se basan en recursos y competencias clave internas, mientras que los negocios de exploración tienen sus cimientos en la visión externa de las oportunidades que brinda el mercado (Cortimiglia et al., 2016).

\section{El enfoque sustentable de los BM}

En este enfoque se afirma que los objetivos comerciales se fortalecen cuando la sostenibilidad se integra como un valor primario. En los artículos clasificados bajo este enfoque (Tabla 1) se distinguen dos corrientes: social y ambiental. La corriente social enfatiza que, con el ánimo de atraer y mantener el interés de los inversionistas sociales, se deben especificar los objetivos de ganancia social de manera clara y temprana y experimentar continuamente con los BM (Yunus et al., 2010). Sin embargo, la mayoría de organizaciones aún no logran desarrollar procesos de creación de valor bajo estos parámetros (Mangematin et al., 2017). Con el fin de subsanar esta deficiencia se aconseja combinar el modelo Canvas con herramientas para el desarrollo estratégico sustentable, tales como: técnicas de creatividad, mapeo de redes de valor y evaluación del ciclo de vida de los productos y servicios. Esta mezcla no solo puede apoyar el diseño y la innovación del modelo de negocio para un desarrollo estratégico sustentable sino que también puede contribuir al crecimiento de los negocios a nivel global (França et al., 2017).

La estrategia colectiva, definida por Astley y Fombrun (1983) como la formulación conjunta de políticas y la implementación de acciones por parte de los miembros de las colectividades organizacionales, toma un papel relevante en la corriente social (Massa et al., 2017) buscando cumplir con objetivos sociales sin sacrificar los económicos. Esto ha propiciado el florecimiento de los BM híbridos (Manning, 2017; Barki et al., 2015), dentro de los que resaltan los BM inclusivos que contribuyen a la reducción de la pobreza y se caracterizan por emplear a personas de poblaciones vulnerables para desarrollar productos y servicios ajustados a las necesidades sociales de sus comunidades (Thompson y MacMillan, 2010). De igual manera, se han desarrollado BM colaborativos que involucran la actuación de empresas sociales locales pioneras. Los BM colaborativos conciben a la población de base de la pirámide (BoP) como clientes potenciales, en lugar de ser vistos como agentes receptores de donaciones. Para atender estas comunidades las organizaciones trabajan en armonía con pequeñas organizaciones locales que cuentan con recursos tangibles (tecnología a bajo costo, acceso a fondos de desarrollo), intangibles (confianza y reputación) y capacidades (de gestión y experiencia). Su propósito es abastecerse de un potencial estratégico que podría resultar difícil o tardar mucho tiempo en construirse de manera aislada (Seelos y Mair, 2007). De este modo se ha cambiado el enfoque paradigmático de los BM orientados a las transacciones hacia un enfoque a la participación, que incluye la colaboración con instituciones de conocimiento, instituciones gubernamentales y empresas sociales, y la construcción de compromiso a nivel comunitario (Goyal et al., 2015).

Dentro de la corriente ambiental, las nociones de ecología industrial y metabolismo industrial sirven de guía, ya que facilitan la comprensión de la forma en que los BM se pueden orientar hacia una economía circular. Lo anterior implica el diseño de negocios que impulsan la reutilización de desechos, incentivan el reciclaje y contribuyen a reducir las emisiones de efecto invernadero sin sacrificar el crecimiento económico (Bocken et al., 2016). Para cumplir con dichos objetivos, se propone el desarrollo de estrategias encauzadas hacia la 
ralentización de los bucles de recursos (reutilización de recursos) y ampliación del periodo de utilización de los productos (mantenimiento y mejora de los productos comprados por el cliente).

\section{El enfoque integrador de los BM}

Los estudios integradores (Tabla 1) se centran en analizar los procesos de configuración (cambios en la estructura) y transformación (cambios en la estrategia) de los negocios, siendo la estrategia un plan sobre cómo debe configurarse el BM. Así, la estrategia no solo define qué BM se empleará, sino también qué patrón de innovación se requiere, dependiendo de las contingencias. En el proceso de formulación de la estrategia, el diseño y mejoramiento del BM se sitúa en la etapa de implementación de alternativas estratégicas. En este enfoque, el BM es un marco para la ejecución de la estrategia y fuente de ventaja competitiva sostenible a través de una innovación continua y eficiente (Cortimiglia et al., 2016).

Si bien los BM son el resultado de una estrategia planificada, su configuración también depende de las contingencias del entorno, las capacidades de la empresa, su experiencia internacional, su dependencia del mercado exterior y su dispersión geográfica. Por ejemplo, para el caso de las multinacionales, algunas prefieren un BM local para cada producto y mercado en el que compiten, otras optan por un BM con características similares en aquellos mercados donde cuentan con participación y unas más desarrollan un BM productivo transferible a otros mercados regionales o extranjeros (Tallman et al., 2018). Sin embargo, el éxito de una organización no solo depende del diseño de su BM, además, se deriva de una apropiada gestión del mismo en relación con sus competidores (Brea-Solís et al., 2015). Dentro de este marco de ideas, el modelo de negocio cumple dos propósitos interrelacionados: proporcionar cierta estabilidad para el desarrollo de las actividades y ser lo suficientemente flexible como para permitir los cambios. Estos se incorporan por fases: creación, extensión, revisión y finalización. La creación enmarca la conceptualización e implementación de un nuevo BM; la extensión implica expandir el negocio sin afectar los procesos existentes dentro del BM; la revisión incorpora cambios fundamentales a las prácticas de trabajo existentes y; la terminación se refiere al abandono del negocio o eliminación de procesos. Sin embargo, la inercia organizativa, los patrones de dependencia, las manifestaciones cognitivas y los problemas políticos y de poder son limitantes que impiden la implementación de los cambios necesarios para ajustar los BM a las condiciones del entorno. (Cavalcante et al., 2011).

\section{Panorama y futuras líneas de trabajo}

De los 14 trabajos identificados como pioneros en la revisión literaria (Tabla 1), el 57,1\% se centra en el enfoque prescriptivo, con temas relacionados con el significado y funcionamiento de los BM. Un $21,4 \%$ se orienta hacía el enfoque del aprendizaje, resaltando la innovación en los BM (Chesbrough, 20010; 2006), la transferencia de conocimiento entre empresas (Mason y Leek, 2008) y el riesgo derivado de la gestión de los negocios duales (Markides y Charitou, 2004). El 14,3\% de los trabajos, dirige su atención hacía el enfoque contextual, con temas que guardan relación con la influencia de la tecnología en innovación de los BM (Chesbrough y Rosenbloom, 2002). También comienza a ser visible el enfoque sustentable con un $7,1 \%$ de participación, donde se presentan los BM como una oportunidad para equilibrar los beneficios económicos y sociales (Thompson y MacMillan, 2010). Los enfoques cognitivo, y cultural se manejan de manera complementaria, con una participación del 35,7\% y 14,3\% respectivamente. El rango cronológico de dichos estudios oscila entre los años 2001 y 2010 , con un 35,7\% de concentración en el año 2010, situación que puede ser motivada por la publicación de la edición especial sobre significado y funcionamiento de los BM, presentada en ese mismo año por la revista Long Range Planning, la cual impulsó la investigación en el campo de los BM al agrupar en un solo número varios estudios de autores pioneros.

Dentro de los 16 trabajos clasificados como extensiones de los pioneros (Tabla 1), se distinguen los enfoques del aprendizaje $(37,5 \%)$ y contextual $(25 \%)$. El enfoque del aprendizaje se robustece con temas sobre la evolución de las capacidades dinámicas (Achtenhagen et al., 2013); la innovación del BM como capacidad dinámica (Christensen, 2016), la evolución ecológica de los negocios (0’Reilly III et al., 2009) y el aprendizaje de los negocios (Andries et al., 2013; Cortimiglia et al., 2016). El enfoque contextual se fortalece con contenidos de investigación, tales como: la perspectiva determinista de los negocios (Zott y Amit, 2008) y el ajuste estratégico de los negocios (Demil et al., 2015; Smith et al., 2010). El enfoque cognitivo tiene una participación del 12,5\%, incluyendo temas tales como: el enfoque sicológico de los negocios (Martins et al., 2015) y la lógica dominante de los BM (Baden-Fuller, 2013).

El grado de atención hacia el enfoque prescriptivo disminuye a un $12,5 \%$ y los temas centrales de investigación son el significado y el funcionamiento de los BM desde una orientación estratégica. Los enfoques sustentable e integrador representan una pequeña participación (6,25\% cada uno de ellos). De estas perspectivas se puede decir que el primero de ellos, trata asuntos relacionados con la responsabilidad social empresarial-RSE (Yunus et al., 2010) y el segundo se concentra en la transformación de los negocios 
(Cavalcante et al., 2011). También se exponen brevemente, como principales vertientes complementarias, los enfoques cognitivo $(33,3 \%)$ contextual $(25 \%)$ y del aprendizaje $(25 \%)$; en menor proporción se identifican la prescriptiva $(8,3)$ e integradora $(8,3 \%)$. Los trabajos de este grupo que tratan de manera exclusiva los enfoques centrales (63\%), fueron publicados a partir del año 2013 y refieren gran parte de los estudios pioneros.

En el grupo conformado por los 21 estudios sobre BM que son calificados como nuevas ramificaciones (Tabla 1), se exponen los enfoques centrales de investigación con mayor grado de especificidad. El enfoque sustentable, con un $28,6 \%$ de participación, se consolida con corrientes de investigación en el campo de los BM sociales y ambientales. En la primera corriente, se exponen temas, tales como: los BM híbridos e inclusivos (Barki et al., 2015; Goyal et al., 2015) y técnicas para el diseño y desarrollo de BM sociales (Franca et al., 2017). De la segunda corriente, se puede inferir que es levemente visible y que además de enfocarse en proyectos sobre BM que reducen el impacto ambiental, se presenta como una nueva orientación de investigación que tiene que ver con la ecología industrial. Otro de los enfoques que presentan nuevas orientaciones de investigación es el cognitivo (19\%), que abarca el estudio de las características del directivo y la lógica de los negocios (Antoldi et al., 2016; Ocasio y Radoynovska, 2016; Saebi y Foss, 2015).

El enfoque contextual (14,3\%), incluye el papel de los activos incumbentes (Kim y Min 2015) y las estrategias de transición (Teymoori et al., 2016). El enfoque del aprendizaje (14,3\%) introduce el tema de la estrategia como práctica (Stefanovic y Milosevic, 2012) y el rol simbólico de los BM (Hacklin y Wallnöfer, 2012). El enfoque prescriptivo $(9,5 \%)$, enfatiza en las opciones tácticas (Haubro et al., 2015) y las técnicas y herramientas estratégicas para la evaluación de los negocios (Bouwman et al., 2018). El enfoque integrador (9,5\%), aborda la gestión de los BM y las transformaciones estructurales (Tallman et al., 2018; Brea-Solis et al., 2015). Por último, la cultural $(4,8 \%)$, se encauza hacía la cultura creativa. Como perspectivas complementarias de estos trabajos se identifican las perspectivas del aprendizaje (50\%), prescriptiva (16,7\%), cognitiva $(16,7 \%)$,) e integradora (16,7\%). Con el ánimo presentar un panorama solido que brinde una visión holística sobre las corrientes de investigación que se abordan desde los diferentes enfoques propuestos en esta revisión. En la figura 2 se presenta una síntesis.

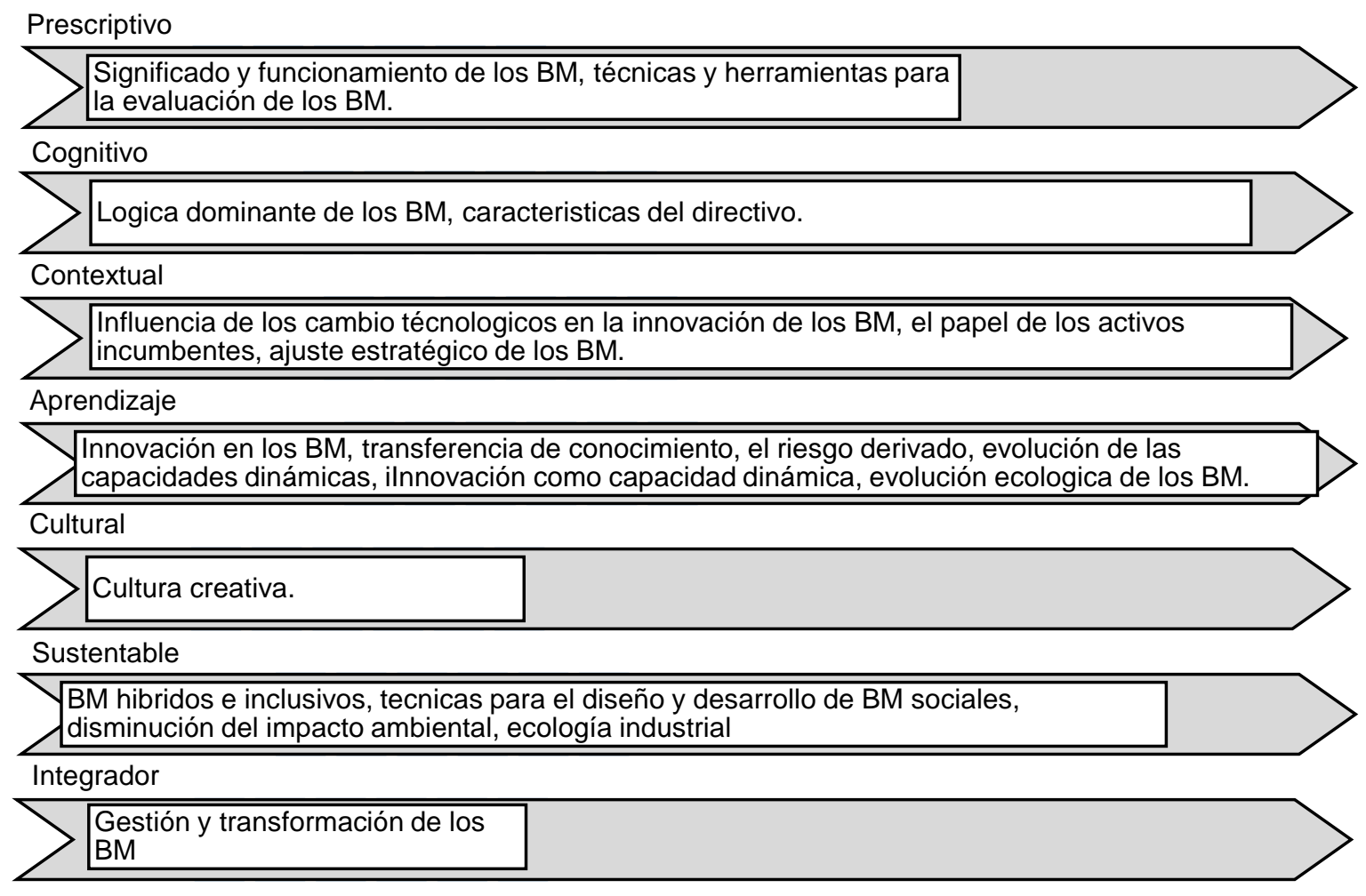

Fig. 2: síntesis de las corrientes de investigación de los enfoques estratégicos de los BM

Aunque los enfoques, prescriptivo, contextual y del aprendizaje han alcanzado un alto grado de madurez, merecen seguir siendo estudiados, considerando que el campo de los BM toma un mayor impulso a partir de año 2010 y se presentan discusiones que continúan latentes. En tal sentido se plantean los siguientes interrogantes: ¿Cómo se crean los BM en diferentes contextos? ¿Cómo las capacidades tacitas de los ejecutivos afectan la capacidad de responder al cambio?; También, se propone validar empíricamente las técnicas y herramientas de evaluación de los BM, estudiar el impacto de las actividades de innovación en los $\mathrm{BM}$, profundizar en las capacidades críticas para la creación de valor que impulsan el desarrollo y el cambio 
de los BM y establecer comparaciones empíricas entre la estrategia formal y emergente con el fin de entender las diferencias en términos de procesos de innovación en los BM.

Si bien es cierto que el enfoque sustentable ha venido creciendo en importancia desde el año 2015, aún se sigue clamando por investigaciones en este campo, motivo por el cual se proponen las siguientes preguntas de investigación: ¿cuál es el impacto de los nuevos modelos de negocio? ¿Qué tipo de transformación producen? ¿Qué desafíos sociales pueden abordar los BM? ¿Qué modelos de negocio deberían adoptar las empresas sociales? ¿Cómo hacen los empresarios para hacer modelos sociales de negocios eficientes? En relación con los enfoques cognitivo, cultural e integrador, se propone indagar en los siguientes aspectos: ¿Qué otros procesos cognitivos existen además de los que ya conocemos? ¿En qué medida, factores como la cultura, la posición en el mercado, la centralización, la formalización y el estilo de liderazgo influyen en el proceso de transformación del BM? ¿Cuán homogéneo es el BM en funcionamiento de una multinacional en regiones y países geográficamente diversificados a diferencia de las empresas que operan en el país?

\section{DISCUSIÓN FINAL}

Esta revisión establece un punto de partida para articular las investigaciones, desde la disciplina de la gestión estratégica, alrededor de los enfoques: prescriptivo, cognitivo, contextual, del aprendizaje, cultural, sustentable e integrador, lo cual ofrece una orientación para posicionar iniciativas de investigación que contribuyan a enriquecer la argumentación generada al interior de cada una de estos. Sin embargo al limitar la estrategia de búsqueda sistemática a dos constructos claves "estrategia y BM" con el fin de explorar la prolífica literatura y concentrarse en aquellos artículos que tienen una relación directa con estos temas, se deja de lado estudios que podrían contribuir de manera notable a los diálogos científicos de las diferentes vertientes. Esta situación brinda una oportunidad para que futuras investigaciones, desde una perspectiva metodológica, incorporen búsquedas relacionadas con los planteamientos tratados en el presente estudio. Así que, en línea con enfoque contextual, se podrían incorporar palabras de búsqueda, tales como: ajuste estratégico, disolución ambiental e innovación; de acuerdo con la perspectiva del aprendizaje, se podría profundizar en asuntos relacionados con las capacidades dinámicas, la gestión del conocimiento, el riesgo derivado y los negocios ambidiestros o duales. De igual modo, con el fin de brindar una mayor comprensión sobre la forma cómo se ha ido configurando la literatura alrededor del enfoque sustentable, se requiere profundizar en temas relacionados con el diseño de los negocios híbridos e inclusivos, entre otros.

\section{CONCLUSIONES}

Con base en lo desarrollado en el artículo se concluye que: 1) la clasificación de los enfoques estratégicos en prescriptivo, cognitivo, contextual, del aprendizaje, cultural e integrador brinda los lineamientos básicos para presentar una visión holística sobre los BM y, a su vez, permite explicar las diferencias e intersecciones de la literatura que trata la relación entre estrategia y BM,2) los retos impuestos por las nuevas formas de hacer negocios, motivan el surgimiento de otras perspectivas estratégicas, como es el caso del enfoque sustentable, que emerge por el compromiso de impulsar el desarrollo económico y social, sin poner en riesgo los recursos a futuro. 3) el estudio interdisciplinario de los BM aumenta el nivel de madurez de los enfoques; en ese sentido, los enfoques cognitivo y cultural deben integrar conocimientos desde las disciplinas de la psicología y la sociología para avanzar en la investigación. 5) las perspectivas estratégicas de los BM se complementan y, aunque los estudios sobre BM se enmarcan dentro de un enfoque particular, resulta conveniente interrelacionarlos para una mayor comprensión del fenómeno a estudiar, lo cual amplia el panorama de investigación. 6) los enfoques estratégicos de los BM presentan diálogos entre los actores sobre los retos que afrontan los negocios actualmente, los cuales ameritan seguirse estudiando para contribuir a la competitividad y sostenibilidad de los mismos.

\section{REFERENCIAS}

Achtenhagen, L., L. Melin y L. Naldi, Dynamics of Business Models-Strategizing, Critical Capabilities and Activities for Sustained Value Creation, doi: 10.1016/j.Irp.2013.04.002, Long Range Planning, 46(6), 427-442 (2013).

Al Humaidan, S. y V. Sabatier, Strategic Renewal in Times of Environmental Scarcity: the Mediating Role of Technology in Business Model Evolution, doi: 10.1108/JOCM-09-2015-0161, Journal of Organizational Change Management, 30(1), 106-120 (2017)

Andries, P., K. Debackere y B. Van Looy, Simultaneous Experimentation as a Learning Strategy: Business Model Development under Uncertainty, doi: 10.1002/sej.1170, Strategic Entrepreneurship Journal, 7(4), 288-310 (2013).

Antoldi, F., E. Capelletti y C. Capelli, Reconsidering the Multi-Sports Club Business Model: Designing Effective New Strategies in the Face of Environmental Changes, doi: 10.1108/MBE-08-2016-0040, Measuring Business Excellence, 20(4), 10-27 (2016).

Amit, R. y C. Zott, Value Creation in E-Business, doi: 10.1002/smj.187, Strategic Management J., 22(6/7), 493-520 (2001). 
Aspara, J., J. A. Lamberg, A. Laukia y H. Tikkanen, Strategic Management of Business Model Transformation: Lessons from Nokia, doi: 10.1108/00251741111126521, Management Decision, 49(4), 622-647 (2011).

Baden-Fuller, C. y M. S. Morgan, Business Models as Models, doi: 1016/j.Irp.2010.03.002, Long Range Planning, 43(2), (2010).

Baden-Fuller, C. y V. Mangematin, Business Models: a Challenging Agenda, doi: 10.1177/1476127013510112, Strategic Organization, 11(4), 418-427 (2013).

Barney, J. B. Firm Resources and Sustained Competitive Advantage, doi: 10.1177/014920639101700108, Journal of Management, 17(1), 99-120 (1991).

Barki, E., G. Comini y otros tres autores, Social Entrepreneurship and Social Business: Retrospective and Prospective Research, ISSN: 2178-938X, Revista de Administração de Empresas, 55(4), 380-384 (2015).

Bock, A. J., T. Opsahl, G. George y D. M. Gann, The Effects of Culture and Structure on Strategic Flexibility During Business Model Innovation, doi: 101111/j.1467-6486.2011.01030.x, Journal of Management Studies, 49(2), 279-305 (2012).

Bocken, N. M., I. De Pauw, C. Bakker y B. Van Der Grinten, Product Design and Business Model Strategies for a Circular Economy, doi: 10.1080/21681015.2016.1172124, Journal of Industrial and Production Engineering, 33(5), 308-320 (2016).

Bouwman, H., J. Heikkilä y otros tres autores, Achieving Agility using Business Model Stress Testing, Electronic Markets, doi: 10.1007/s12525-016-0243-0, 28(2), 149-162 (2018).

Brea-Solís, H., R. Casadesus-Masanell y E. Grifell-Tatjé, Business Model Evaluation: Quantifying Walmart's Sources of Advantage, doi:10.1002/sej.1190, Strategic Entrepreneurship Journal, 9(1), 12-33 (2015).

Casadesus-Masanell, R. y J. E. Ricart, From Strategy to Business Models and onto Tactics, doi: 10.1016/j.Irp.2010.01.004, Long Range Planning, 43(2-3), 195-215 (2010).

Cavalcante, S., P. Kesting y J. Ulhøi, Business Model Dynamics and Innovation: (Re) Establishing the Missing Linkages, doi: 10.1108/00251741111163142, Management Decision, 49(8), 1327-1342 (2011).

Cortimiglia, M. N., A. Ghezzi y A, G. Frank, Business Model Innovation and Strategy Making Nexus: Evidence from a Cross-Industry Mixed-Methods Study, doi: 10.1111/radm.12113, R\&D Management, 46(3), 414-432 (2016).

Christensen, C. M., T. Bartman y D. Van Bever, The Hard Truth about Business Model Innovation, MIT Sloan Management Review, 58(1), 31-40 (2016).

Chesbrough, H. Business Model Innovation: Opportunities and Barriers, doi: 10.1016/j.Irp.2009.07.010, Long Range Planning, 43(2), 354-363 (2010).

Chesbrough, H. W. The Era of Open Innovation, Managing Innovation and Change, 127(3), 34-41 (2006).

Chesbrough, H. y R. S. Rosenbloom, The Role of the Business Model in Capturing Value from Innovation: Evidence from Xerox Corporation's Technology Spin-Off Companies, doi: 10.1093/icc/11.3.529, Industrial and Corporate Change, 11(3), 529-555 (2002).

Demil, B. y X. Lecocq, Business Model Evolution: in Search of Dynamic Consistency, doi: 10.1016/j.Irp.2010.02.004, Long Range Planning, 43(2), 227-246 (2010).

Dyer, J. H. y H. Singh, The Relational View: Cooperative Strategy and Sources of Interorganizational Competitive Advantage, doi: 10.2307/259056, Academy of Management Review, 23(4), 660- 679 (1998).

Díez-Gómez, D., M. Guillén y M. del P. Rodríguez, Toma de Decisiones Éticas en Organizaciones: una Revisión de la Literatura, Información Tecnológica, en prensa, 30(3) (2019).

França, C. L., G. Broman y otros tres autores, an Approach to Business Model Innovation and Design for Strategic Sustainable Development, Journal of Cleaner Production, doi: 10.1016/j.jclepro.2016.06.124, 140, 155-166 (2017).

Hacklin, F., J. Björkdahl y M. W. Wallin, Strategies for Business Model Innovation: how Firms Reel in Migrating Value, doi: 10.1016/j.lrp.2017.06.009, Long Range Planning, 51(1), 82-110 (2018).

Hacklin, F. y M. Wallnöfer, The Business Model in the Practice of Strategic Decision-Making: Insights from a Case Study, doi: 10.1108/00251741211203515, Management Decision, 50(2), 166-188 (2012).

Haubro, A. P., H. A. Lomholt y otros tres autores, Tactical and Strategic Choices in Business Models: Evidence from a Danish Fashion Outlet, Journal of Fashion Marketing and Management, doi: 10.1108/JFMM-07-2014-0056, 19(3), 274289 (2015).

Johnson M. W., C. M. Christensen y H. Kagermann, Reinventing your Business Model, Harvard Business Review, 86(12), 50-59 (2008).

Khanagha, S., H. Volberda y I. Oshri, Business Model Renewal and Ambidexterity: Structural Alteration and Strategy Formation Process during Transition to a Cloud Business Model, doi: 10.1111/radm.12070, R\&D Management, 44(3), 322340 (2014).

Klang, D., M. Wallnöfer y F. Hacklin, The Business Model Paradox: a Systematic Review and Exploration of Antecedents, doi: 10.1111/ijmr.12030, International Journal of Management Reviews, 16(4), 454-478 (2014). 
Kim, S. K. y S. Min, Business Model Innovation Performance: When Does Adding a New Business Model Benefit an Incumbent? doi: 10.1002/sej.1193, Strategic Entrepreneurship Journal, 9(1), 34-57 (2015).

Magretta, J. Why Business Models Matter, Harvard Business Review, 80(5), 86-92 (2002).

Mangematin V., A. M. Ravarini y P. Sharkey Scott, (2017), Practitioner Insights on Business Models and Future Directions, doi: 10.1108/JBS-10-2016-0130, Journal of Business Strategy, 38(2) 3-5 (2017).

Markides, C. y C. D. Charitou, Competing with Dual Business Models: a Contingency Approach, Academy of Management Executive, 18(3), 22-36 (2004).

Martins, L. L., V. P. Rindova y B. E. Greenbaum, Unlocking the Hidden Value of Concepts: a Cognitive Approach to Business Model Innovation, doi: 10.1002/sej.1191, Strategic Entrepreneurship Journal, 9(1), 99-117 (2015).

Mason, K. J. y S. Leek, Learning to Build a Supply Network: an Exploration of Dynamic Business Models, doi.10.1111/j.1467-6486.2008.00769.x, Journal of Management Studies, 45(4), 774-799 (2008).

Massa, L., C. L. Tucci y A. Afuah, A critical Assessment of Business Model Research, doi: 10.5465/annals.2014.0072, Academy of Management Annals, 11(1), 73-104 (2017).

Maucuer, R. y A. Renaud, Business Model Research: a Bibliometric Analisis of Origins \& Trends, Management, Forthcoming (2019).

Mintzberg, H., B. Ahlstrand y J. Lampel, Safari a la Estrategia. Una Visita Guiada por la Jungla del Management Estratégico, Ediciones Granica SA, Buenos Aires (1999).

Morris, M., M. Schindehutte y J. Allen, The Entrepreneur's Business Model: Toward a Unified Perspective, doi: 10.1016/j.jbusres.2003.11.001, Journal of Business Research, 58(6), 726-735 (2005).

Ocasio, W. y N. Radoynovska, Strategy and Commitments to Institutional Logics: Organizational Heterogeneity in Business Models and Governance, doi: 10.1177/1476127015625040, Strategic Organization, 14(4), 287-309 (2016).

O'Reilly III, C. A., J. B. Harreld y M. L. Tushman, Organizational Ambidexterity: IBM and Emerging Business Opportunities, California Management Review, 51(4), 75-99 (2009).

Osterwalder, A. y Y. Pigneur, Business Model Creation, Modderman Drukwerk: Amsterdam, The Netherlands (2009).

Porter, M. E., Competitive Advantage, Free Press, New York (1985).

Priem, R. L., M. Wenzel y J. Koch, Demand-Side Strategy and Business Models: Putting Value Creation for Consumers Center Stage, doi: 10.1016/j.Irp.2017.07.007, Long Range Planning, 51(1), 22-31 (2018).

Robledo, S., G. A. Osorio y C. López, Networking en Pequeña Empresa: una Revisión Bibliográfica utilizando la Teoría de Grafos, ISSN: 1794-211X, Revista Vínculos, 11(2), 6-16, (2014).

Robledo, S., N. Duque y I. Zuluaga, Difusión de Productos a través de Redes Sociales: una Revisión Bibliográfica utilizando la Teoría de Grafos, ISSN: 0122-820X, Respuestas, 18(2), 27-41 (2013).

Saebi, T. y N. J. Foss, Business Models for Open Innovation: Matching Heterogeneous Open Innovation Strategies with Business Model Dimensions, doi: 10.1016/j.emj.2014.11.002, European Management Journal, 33(3), 201-213 (2015).

Seelos, C. y J. Mair, Profitable Business Models and Market Creation in the Context of Deep Poverty: a Strategic View, doi: 10.5465/amp.2007.27895339, Academy of Management Perspectives, 21(4), 49-63 (2007).

Smith, W. K., A. Binns y M. L. Tushman, Complex Business Models: Managing Strategic Paradoxes Simultaneously, doi: 10.1016/j.Irp.2009.12.003, Long Range Planning, 43(2), 448- 461 (2010).

Stefanovic, I. y D. Milosevic, On Conceptual Differentiation and Integration of Strategy and Business Model, Zbornik Radova Ekonomskog Fakulteta u Rijeci: Časopis za Ekonomsku Teoriju i Praksu, 30(1), 141-161 (2012).

Tallman, S., Y. Luo y P. J. Buckley, Business Models in Global Competition, doi: 10.1504/IJGSB.2008.017294, Global Strategy Journal, 8(4), 517-535 (2018).

Teece, D. Business Models, Business Strategy and Innovation, doi: 10.1016/j.Irp.2009.07.003, Long Range Planning, 43(2), 172-194 (2010).

Teymoori, M., E. M. Oskooee y M. R. Khonjeh, Proposing a Transition Strategy Formulation based on Business Model: a Case Study in General Contractor Company, IIOAB Journal, 7, 426-434 (2016).

Thompson, J. D. y I. C. MacMillan, Business Models: Creating New Markets and Societal Wealth, doi: 10.1016/j.Irp.2009.11.002, Long Range Planning, 43(2), 291-307 (2010).

Toro, J. y M. del P. Rodríguez, Formación en Ética en las Organizaciones: Revisión de la Literatura, doi: 10.4067/S071807642017000200018, Información Tecnológica, 28(2), 167-180 (2017).

Wirtz, B. W., A, Pistoia, S. Ullrich y V. Göttel, Business Models: Origin, Development and Future Rresearch Perspectives, doi: 10.1016/j.Irp.2015.04.001, Long Range Planning; 49(1), 36-54 (2016).

Yunus, M., B. Moingeon y L. Lehmann-Ortega, Building Social Business Models: Lessons from the Grameen Experience, doi: 10.1016/j.Irp.2009.12.005, Long Range Planning, 43(2), 308-325 (2010). 
Zott, C., R. Amit y L. Massa, The Business Model: Recent Developments and Future Research, doi: 10.1177/0149206311406265, Journal of Management, 37, 1019-1042 (2011).

Zott, C. y R. Amit, Business Model Design: an Activity System Perspective, doi: 10.1016/j.Irp.2009.07.004, Long Range Planning, 43(2), 216-226 (2010).

Zott, C. y R. Amit, The Fit between Product Market Strategy and Business Model: Implications for firm Performance, doi: 10.1002/smj.642, Strategic Management Journal, 29(1), 1-26 (2008).

Zuluaga, M., S. Robledo y otros cuatro autores, Metabolomics and Pesticides: Systematic Literature Review using Graph Theory for Analysis of References, ISSN: 1794-2470, Nova, 13(25), 7-16 (2016). 